\title{
Regaining Motor Control in Musician's Dystonia by Restoring Sensorimotor Organization
}

\author{
Karin Rosenkranz, ${ }^{1,2}$ Katherine Butler, ${ }^{3}$ Aaron Williamon, ${ }^{4}$ and John C. Rothwell ${ }^{1}$ \\ ${ }^{1}$ Sobell Department of Motor Neuroscience and Movement Disorders and ${ }^{2}$ Department of Clinical and Experimental Epilepsy, UCL Institute of Neurology, \\ London W1N 3BG, United Kingdom, ${ }^{3}$ London Hand Therapy, London W1H 5TF, United Kingdom, and ${ }^{4}$ Royal College of Music, London SW7 2BS, United \\ Kingdom
}

Professional musicians are an excellent model of long-term motor learning effects on structure and function of the sensorimotor system. However, intensive motor skill training has been associated with task-specific deficiency in hand motor control, which has a higher prevalence among musicians (musician's dystonia) than in the general population. Using a transcranial magnetic stimulation paradigm, we previously found an expanded spatial integration of proprioceptive input into the hand motor cortex [sensorimotor organization (SMO)] in healthy musicians. In musician's dystonia, however, this expansion was even larger. Whereas motor skills of musicians are likely to be supported by a spatially expanded SMO, we hypothesized that in musician's dystonia this might have developed too far and now disrupts rather than assists task-specific motor control. If so, motor control should be regained by reversing the excessive reorganization in musician's dystonia. Here, we test this hypothesis and show that a 15 min intervention with proprioceptive input (proprioceptive training) restored SMO in pianists with musician's dystonia to the pattern seen in healthy pianists. Crucially, task-specific motor control improved significantly and objectively as measured with a MIDI (musical instrument digital interface) piano, and the amount of behavioral improvement was significantly correlated to the degree of sensorimotor reorganization. In healthy pianists and nonmusicians, the SMO and motor performance remained essentially unchanged. These findings suggest that the differentiation of SMO in the hand motor cortex and the degree of motor control of intensively practiced tasks are significantly linked and finely balanced. Proprioceptive training restored this balance in musician's dystonia to the behaviorally beneficial level of healthy musicians.

\section{Introduction}

Intensive motor training induces structural and functional changes in the brain (Kleim et al., 2004; Rosenkranz et al., 2007b) but has also been associated with the development of focal hand dystonia, a task-specific disorder of hand motor control (Byl et al., 1996). Professional musicians are an excellent human model for long-term motor training, since most of them started playing at a very early age and the structural and functional changes observed in their brains depend on the age at which their musical training commenced (Gaser and Schlaug, 2003; Bengtsson et al., 2005; Rosenkranz et al., 2007a). Interestingly, the prevalence of focal hand dystonia among professional musicians (musician's dystonia) is higher than in the general population (Nutt et al., 1988; Altenmüller, 2003). Although some experimental findings in focal hand dystonia (Stinear and Byblow, 2004; Quartarone et al., 2006) are similarly observed in healthy musicians (Rosenkranz et al., 2007a) and therefore do not distinguish pathological from physiological reorganization, others show that structural and functional brain reorganization progressed from healthy musicians to musicians affected with focal hand dystonia (Elbert et al.,

Received May 4, 2009; revised Sept. 21, 2009; accepted Sept. 24, 2009.

This work was supported by the Dystonia Medical Research Foundation, the Bachmann-Strauss Dystonia \& Parkinson Foundation, and the Medical Research Council.

Correspondence should be addressed to Dr. Karin Rosenkranz, Department of Clinical and Experimental Epilepsy, UCL Institute of Neurology, 33 Queen Square, London W1N 3BG, UK. E-mail: k.rosenkranz@ion.ucl.ac.uk. DOI:10.1523/JNEUROSCI.2094-09.2009

Copyright $\odot 2009$ Society for Neuroscience ～0270-6474/09/2914627-10\$15.00/0
1995, 1998; Rosenkranz et al., 2005). One sensitive parameter is the integration of experimentally induced proprioceptive input from the hand muscles in the motor cortex [sensorimotor organization (SMO)], which we developed in previous studies (Rosenkranz and Rothwell, 2003). While healthy nonmusicians show a characteristic differential pattern of SMO, with reduced intracortical inhibition in projections to the vibrated muscle and increased intracortical inhibition in "surrounding" projections to the nonvibrated ones, this pattern was less well differentiated in healthy musicians and lost in musician's dystonia (Rosenkranz et al., 2005). Given the importance of proprioceptive input for motor learning (Pavlides et al., 1993), the changes observed in healthy musicians are likely to have developed during musical skill learning and to support performance at the highest level. However, in musician's dystonia, this reorganization might have gone too far such that it interferes with motor control rather than assists it (Rosenkranz et al., 2005).

If this association between the amount of sensorimotor reorganization and the level of motor control holds true, reversing the excessive reorganization in musician's dystonia toward the pattern seen in healthy musicians should reestablish motor control.

We have shown previously that a 15 min intervention with proprioceptive stimulation, which required the subjects' attention to be focused on the vibration, changes the SMO in musician's dystonia and makes it more similar to that seen in healthy musicians spontaneously (Rosenkranz et al., 2008). Here, we crucially expand these findings by investigating whether restoring a 
more differential pattern of SMO by proprioceptive stimulation influences motor control of the affected task in musician's dystonia. We used a modified version of our previous proprioceptive training paradigm and applied vibration randomly to three hand muscles rather than the thumb abductor alone, which further strengthen the differentiating effect on SMO. We examined pianists with focal hand dystonia compared with healthy pianists, to avoid confounds by specific brain changes induced by long-term training of different musical instruments (Bangert and Schlaug, 2006).

\section{Materials and Methods}

\section{Subjects}

Six healthy musically naive subjects (mean age, 34 years), eight healthy professional pianists (mean age, $31 \pm 2$ years) (for details, see supplemental Table 1, available at www.jneurosci.org as supplemental material), and eight professional pianists with musician's dystonia (mean age, $33 \pm 3$ years) (for details, see supplemental Table 2, available at www. jneurosci.org as supplemental material) were studied. Groups were matched for age; in addition, healthy and dystonic pianists were matched for age at which piano playing was started and daily practice time. All pianists with musician's dystonia had task-specific symptoms that exclusively occurred during piano playing and involved predominantly the ring finger of the right hand ("pulling-in") when playing downward scale-like movements. None of the patients had received botulinum toxin injections in the hand/forearm muscle before, which could affect neurophysiological findings. All subjects gave informed consent to the study, which was approved by the local ethics committee and conformed to the Declaration of Helsinki.

\section{Transcranial magnetic stimulation and}

\section{electromyographic recording}

Transcranial magnetic stimulation (TMS) was performed using two MAGSTIM 200 stimulators connected by a Y-cable to a figure-of-eightshaped coil with an internal wing diameter of $7 \mathrm{~cm}$ (Magstim). The coil was held with the handle pointing backward and laterally $\sim 45^{\circ}$ to the interhemispheric line to evoke anteriorly directed current in the brain and was optimally positioned to obtain motor-evoked-potentials (MEPs) in abductor pollicis brevis (APB) muscle as the main target muscle. The active motor threshold ( $\mathrm{aMT}$ ) defined as the minimum intensity needed to evoke a MEP of $>200 \mu \mathrm{V}$ in 5 of 10 trials was measured in the tonically active APB ( $\sim 20 \%$ of maximal contraction as assessed visually on an oscilloscope). Stimulation intensities are quoted in the text as a percentage of maximal stimulator output $( \pm \mathrm{SE})$.

Surface electromyographic (EMG) recordings in a belly-to-tendon montage were made from APB, first dorsal interosseus (FDI), and abductor digiti minimi (ADM). The raw signal was amplified and filtered (30 $\mathrm{Hz}$ to $1 \mathrm{kHz}$ ) (Digitimer). Signals were digitized at $2 \mathrm{kHz}$ (CED Power1401; Cambridge Electronic Design) and stored on a laboratory computer for off-line analysis.

\section{Proprioceptive training}

Proprioceptive training lasted for $15 \mathrm{~min}$ and involved repeated cycles of muscle vibration ( $2 \mathrm{~s}$ on, $2 \mathrm{~s}$ off) applied in equal amounts to either the $\mathrm{APB}, \mathrm{FDI}$, or ADM muscles in a truly random order by using electromagnetic mechanical stimulators (Ling Dynamics System) with a 0.7-cmdiameter probe. These stimulators were mounted on an armrest on which the subjects rested their right pronated forearm. The probe of each stimulator was positioned orthogonally to, and under slight pressure against, the belly of the hand muscle between the EMG electrodes. The amplitude $(0.2-0.5 \mathrm{~mm})$ of the vibration was adjusted individually to be just below threshold for perceiving an illusory movement (Gilhodes et al., 1986; Roll and Gilhodes, 1995)

Subjects were asked to focus their attention on the vibrated muscle and to discriminate subtle changes of vibration frequency occurring during the vibration period. In $75 \%$ of trials at random, the frequency of the vibration was changed from 80 to $67.5,72.5$, or $77.5 \mathrm{~Hz}$ for the last $300 \mathrm{~ms}$ of the $2 \mathrm{~s}$ train. In the $2 \mathrm{~s}$ rest period between vibration, subjects had to

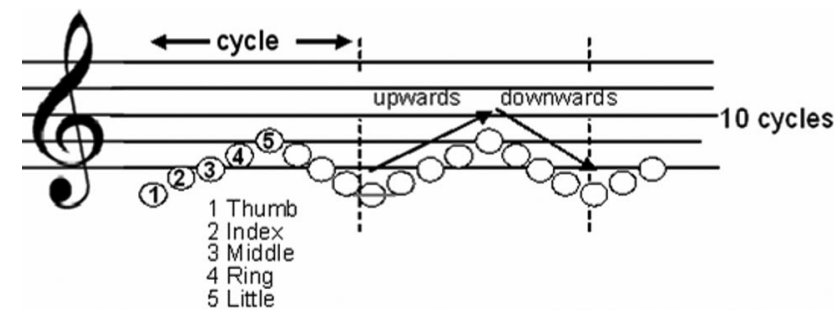

Figure 1. Five-finger exercise performed on a MIDI piano (Yamaha Clavinova CLP170). This involved playing the first five notes of the $(\mathrm{C}$ major scale using all five fingers of the right hand, starting with the thumb to the little finger and vice versa. Ten cycles were performed following a metronome set at $200 \mathrm{bpm}$ ( 1 note per beat). Two different levels of loudness (pianissimomezzoforte) and musical notation (staccato-legato) were tested. The loudness level was verified by comparing the velocity of key press, which was not significantly different between groups. Furthermore, staccato and legato were verified, respectively, by the absence or presence of overlay between successive notes.

report whether they perceived a change or not by pressing buttons on a response box with their left hand. They were instructed to be as accurate rather than as quick as possible. After each trial auditory feedback was given of whether or not their answer was correct.

\section{Experimental parameters}

Sensorimotor organization. The sensorimotor organization (SMO) was measured following previously described protocols (Rosenkranz and Rothwell, 2003, 2006a,b; Rosenkranz et al., 2005, 2008). In brief, single (test pulse alone) or pairs of pulses (conditioning and test pulse; interstimulus interval, $3 \mathrm{~ms}$ ) (Kujirai et al., 1993) to measure the shortinterval intracortical inhibition (SICI) were applied randomly every $5 \mathrm{~s}$. The intensity of the test stimulus was set to evoke an MEP of $\sim 1 \mathrm{mV}$ peak-to-peak amplitude, and the subthreshold conditioning stimulus was set to evoke a $50 \%$ inhibition of the test MEP $(\sim 80 \%$ aMT). On one-quarter of trials each, stimuli were applied either in the presence of APB vibration (vibAPB), FDI vibration (vibFDI), ADM vibration (vibADM), or without vibration. Muscle vibration was applied in trains of $1.5 \mathrm{~s}$ duration, followed by a $3.5 \mathrm{~s}$ vibration-free interval (total trial length, $5 \mathrm{~s}$ ) using the same parameters and probe positions as mentioned above, which did not induce any vibration artifact in the EMG trace. The TMS single pulse or test pulse was applied every $5 \mathrm{~s}$ and was timed to appear $1 \mathrm{~s}$ after the onset of vibration. During vibration, EMG was monitored for any muscle contraction indicating, besides possible voluntary activation, the occurrence of the tonic vibration reflex (Hagbarth and Eklund, 1968; Marsden et al., 1969). A total of 80 trials were collected with 10 trials of each condition.

Although measurement of SICI involves recording MEPs in response to single-pulse TMS, we have not reported on these findings in detail in the present study; instead, we focused on reporting SICI that represents $\mathrm{GABA}_{\mathrm{A}}$ receptor-mediated inhibition (Müller-Dahlhaus et al., 2008) and is a better measure of the purely cortical effect of proprioceptive training (DiLazzaro et al., 1998).

It should be noted that although short-term vibration increases the amplitude of the test MEP, we have previously shown that the percentage of SICI during vibration is unaffected by variations in MEP amplitude over this range in healthy subjects, healthy musicians, and patients with musician's dystonia (Rosenkranz and Rothwell, 2003; Rosenkranz et al., 2005). Thus, it was not necessary to adjust the test pulse intensity to evoke MEPs of matching size ( $1 \mathrm{mV}$ peak-to-peak amplitude).

Piano performance test. To objectively evaluate the piano performance, subjects were asked to play 10 cycles of a five-finger exercise (Fig. 1) on a musical instrument digital interface (MIDI) piano (Yamaha Clavinova CLP170) following a metronome set at $200 \mathrm{bpm}$ (one note per beat). Two different levels of loudness (pianissimo-mezzoforte) and musical notation (staccato-legato) were tested for the right hand. The loudness level was verified by comparing the velocity of key press, which was not significantly different between groups. Furthermore, staccato and legato were verified by the absence or presence of overlay between successive notes, respectively. Healthy nonmusician subjects were given $10-15 \mathrm{~min}$ 

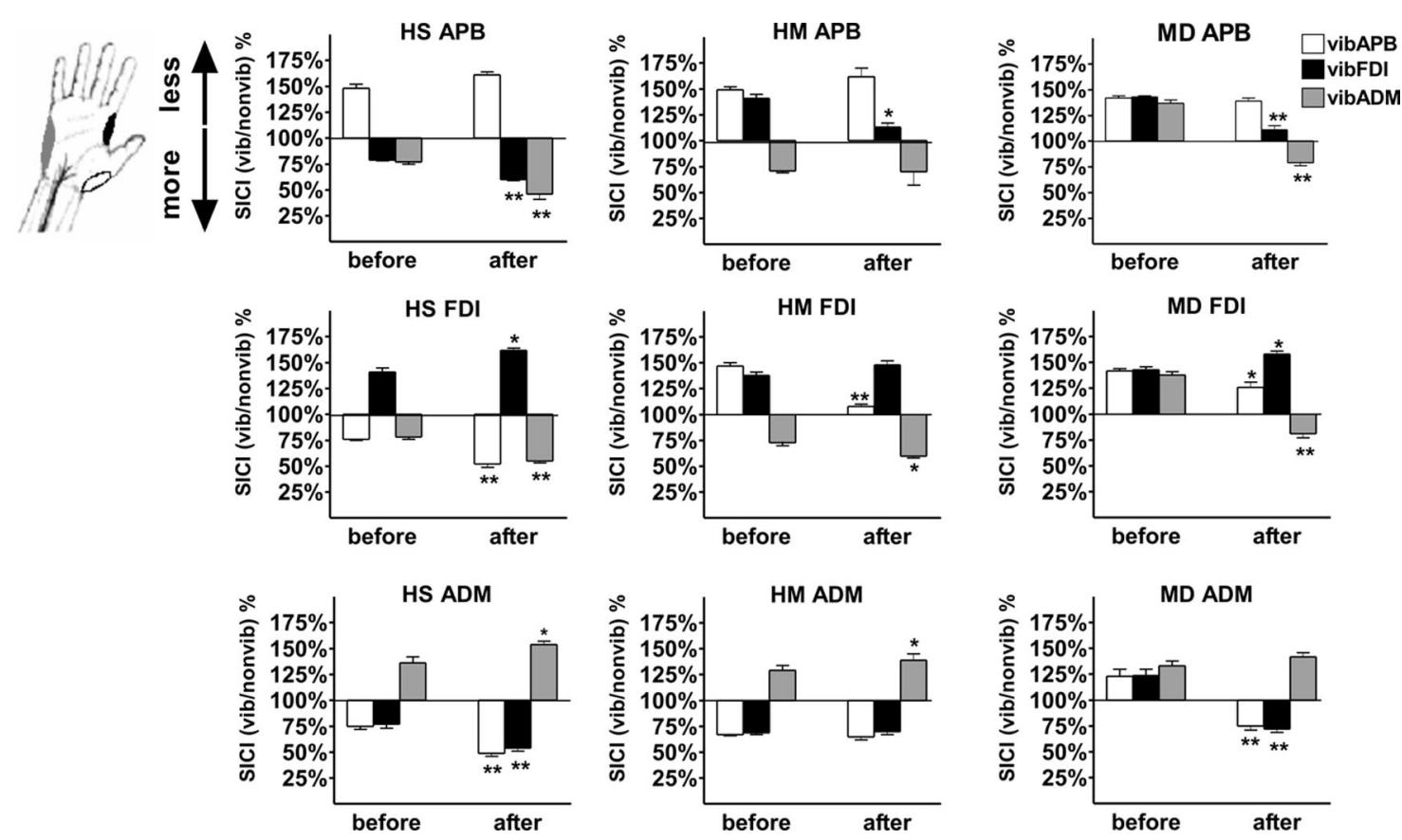

Figure 2. SICl during vibration (vib) as a percentage of the SICl obtained without vibration (nonvib) ( \pm SE). The three columns display data for HS, HM, and MD, whereas the rows show SICI recorded in the three different hand muscles (APB, FDI, ADM), before and after proprioceptive training. The bars show the normalized SICI with vibAPB (white bars), vibFDI (black bars), or vibADM (gray bars). Decreases of SICl are plotted as columns going up (i.e., less percentage inhibition of the test response), and increases are plotted as columns going down. Statistical results of paired $t$ tests comparing baseline data (before) with data obtained after proprioceptive training are shown as asterisks (with ${ }^{*} p \leq 0.05$; $^{* *} p \leq 0.001$ ).

tuition on the task until their performance was stable. All healthy and dystonic pianists were given time to familiarize themselves with the particular mechanics of this piano. The duration of the key press was measured for each individual finger during the cycle, distinguishing between upward (thumb to little finger) and downward (little finger to thumb) movements. The coefficient of variance (CoVar) for the duration of key press was calculated as a measure of performance variability. To give an additional simple summarizing measure the mean CoVar (duration) averaged over all finger movements was calculated for each subject.

Self-assessment of piano performance. All participants were asked to express on a visual-analog scale (VAS) whether they felt their performance of the five-finger exercise on the piano to be better or worse than before the proprioceptive training [VAS (change in performance)] (supplemental Fig. $1 \mathrm{~A}$, available at www.jneurosci.org as supplemental material). For analysis, the items were translated into a score with 0 indicating "no change" and 1-3 describing that the performance was "slightly," "moderately," or "strongly" changed with positive/negative values indicating improvement/worsening. Additionally, musician's dystonia patients were asked to rank their level of impairment on a VAS (impairment) scale ranging from "none" to "absolute" before and directly after each performance of the five-finger exercise. For analysis, the items were scaled from 0 (none) to 6 (absolute) (supplemental Fig. $1 B$, available at www.jneurosci.org as supplemental material).

Clinical evaluation. Whereas the assessment of piano performance was based on the objectively assessed performance on the MIDI piano, hand motor control was additionally assessed before and after proprioceptive training using the Burke-Fahn-Marsden (BFM) Scale and TubianaChamagne Scale (TCS) (Tubiana and Chamagne, 1993) (supplemental Table 3, available at www.jneurosci.org as supplemental material) for reasons of comparability to other clinically oriented studies on hand dystonia.

Vibration discrimination data. During the proprioceptive training, subtle changes of vibration frequency had to be discriminated (see above). These behavioral data were digitized and stored on the computer for off-line analysis, and the number of errors performed per condition (frequency interval) and muscle were calculated.
Protocol

At the start of the experiments, all participants performed the five-finger exercise on the MIDI piano, after which the patients were asked to subjectively assess their level of impairment on the VAS (impairment). Then, the participants were prepared for the TMS experiment, and baseline SMO was recorded. Following this, proprioceptive training was performed for $15 \mathrm{~min}$. After a break of $10 \mathrm{~min}$, the SMO was recorded again. After an additional break of 15 min during which the EMG electrodes were removed, the participants were asked to perform the piano exercise again.

All participants then rated their performance on the VAS (change of performance), and additionally, musician's dystonia patients rated their performance on the VAS (impairment). The patients were then asked to continue playing at home some repertoire of their choice, to repeat the five-finger exercise and their self-rating via VAS (impairment) 6 and $24 \mathrm{~h}$ after the end of proprioceptive training and to report back via telephone (for an illustration of the experimental protocol and session time line, refer to supplemental Fig. 2, available at www.jneurosci.org as supplemental material).

\section{Data analysis and statistics}

The subjects' age and TMS parameters, the vibration discrimination data, and the VAS (change of performance) scores were compared between all groups, and the clinical scores (BFM/TCS) and VAS (impairment) were compared in musician's dystonia before/after training by use of ANOVA and parameteric ( $t$ tests) or nonparametric (Wilcoxon's) tests where necessary (see Results for detail).

Neurophysiological and piano performance data. For data analysis and presentation, the neurophysiological data was simplified. After establishing that the MEPs obtained in all hand muscles without vibration before and after the intervention were not significantly different (paired $t$ tests), the amount of SICI measured during vibration of APB, FDI, or ADM was expressed as a percentage of SICI without vibration. Statistical analysis was performed on this normalized SICI (Fig. 2).

ANOVA was used for the statistical analysis of the neurophysiological and piano performance data, followed by $t$ tests where necessary. The factors used for the analysis of the neurophysiological data were TRAINING 
Table 1. Subjects' age and TMS parameters defined in the APB ( \pm SE)

\begin{tabular}{lrlll}
\hline & \multicolumn{1}{c}{ Age (years) } & aMT & Conditioning SI & Test SI \\
\hline Healthy subjects & $34.0 \pm 2.3$ & $36.7 \pm 3.4$ & $29.8 \pm 2.7$ & $56.2 \pm 5.6$ \\
Healthy musicians & $31.43 \pm 2.3$ & $32.8 \pm 1.7$ & $26.4 \pm 0.9$ & $54.8 \pm 3.6$ \\
$\begin{array}{l}\text { Musician's dystonia } \\
\text { ANOVA (GROUP) }\end{array}$ & $33.0 \pm 2.5$ & $35.0 \pm 1.9$ & $29.0 \pm 1.5$ & $54.5 \pm 3.9$ \\
$F_{(2,18)}$ & 1.12 & 0.52 & 0.81 & \\
$p$ & 0.37 & 0.59 & 0.48 & 0.10 \\
\hline
\end{tabular}

TMS parameters are given in percentage stimulator output. SI, Stimulus intensity.

(before/after training), MUSCLE (APB, FDI, ADM), and VIBRATION CONDITION (vibAPB, vibFDI, vibADM). The analysis of the piano performance data was performed for the two conditions (mezzoforte, pianissimo) separately on the parameters duration and CoVar (duration) with the factors TRAINING and FINGER MOVEMENT.

The between-group statistics on neurophysiological and behavioral data could be confounded by the baseline differences between the groups, especially when including the factor TRAINING. Therefore, the between-group analysis was performed on the data obtained either before or after the proprioceptive training. To minimize this confound further, we performed two different analyses involving the factor GROUP, one including the groups of healthy subjects and healthy musicians (HS/HM) only and another including healthy musicians and musician's dystonia (HM/MD).

All ANOVAs with repeated-measures design were tested for sphericity using Mauchly's test. In case of significant sphericity, GreenhouseGeisser corrections were performed. Corrected ANOVAs are marked with an asterisk $\left({ }^{*}\right)$.

Correlation of SMO and piano performance data in musician's dystonia. To get a summarizing measure of the changes of SMO after proprioceptive training (SMOchange) the difference (after - before proprioceptive training) of the normalized SICI data (vibrated/nonvibrated; as shown in Fig. 2) were calculated. To provide a single value for the homotopic (effect of vibration on the vibrated muscle), the near heterotopic (effect of vibration on the nearby nonvibrated muscle; e.g., vibAPB on FDI, vibFDI on $\mathrm{APB}$ ), and far heterotopic (effect of vibration on the far nonvibrated muscle; e.g., vibADM on APB or FDI) effects, the data obtained in APB and FDI were averaged. The distinction between near and far heterotopic effects was made since the effect of proprioceptive training on the "far" heterotopic effect was expected to be stronger than that on the "near" heterotopic effect. The data obtained in the ADM was not included, since both heterotopic effects induced by vibAPB and vibFDI could be considered as far and therefore would have contributed an unequal amount of observations.

The averaged SMOchange was correlated with the difference (after before proprioceptive training) of the mean key press duration (DURchange) and CoVar (COVARchange) for each finger movement in the mezzoforte and pianissimo condition separately. The $r^{2}$ values were calculated, and statistical analysis was performed using $t$ tests.

The significance level was set at $p \leq 0.01$ for ANOVAs to correct for multiple comparisons, and to $p \leq 0.05$ for $t$ tests and Wilcoxon's test. All data are given as means \pm SE.

\section{Results}

\section{Subjects' and TMS parameters}

The mean age, the aMT, and the stimulus intensities used for test and conditioning pulses were not different between the groups [ANOVA (GROUP); $F_{(2,18)}>0.07 ; p>0.3$ ] (Table 1). The age at which instrumental playing started and the amount of actual daily playing were similar in healthy musicians and musician's dystonia ( $t$ test $p=0.6$ for starting age; $p=0.8$ for practice time). Furthermore, in all groups, the amplitudes of the test MEPs and SICI recorded in all muscles without vibration before and after proprioceptive training were similar [ANOVA (GROUP); $F_{(2,18)}<$ $0.071 ; p>0.09]$, thus allowing for a simplification of the data set as described above (see Materials and Methods).

\section{Neurophysiological data}

Baseline SMO

Figure 2 shows SICI during vibration as a percentage of the amount of SICI obtained without vibration for each group before and after proprioceptive training. Decreases of SICI (i.e., less inhibition) are plotted as columns going up, and increases are plotted as columns going down.

At baseline, in healthy subjects muscle vibration reduced SICI in the vibrated muscle, while having the opposite effect on nonvibrated muscles. This pattern was less distinctive in healthy musicians. Here, vibration of either APB or FDI reduced SICI in both FDI and APB, while still increasing SICI in ADM. However, vibration of ADM did enhance the SICI in the nonvibrated muscles. In musician's dystonia, vibration of one muscle reduced SICI in all recorded muscles. These results confirm our previous findings obtained in different groups of patients (Rosenkranz et al., 2005, 2008).

A within-group two-way ANOVA with the factors MUSCLE and VIBRATION CONDITION showed a significant interaction in healthy subjects $\left(F_{(4,20)}=498.8 ; p<0.0001\right)$ and healthy musicians $\left(F_{(4,20)}=314.64 ; p<0.0001\right)$, but not in musician's dystonia. This indicates that in the latter, vibration of any muscle had a similar effect on SICI recorded in all muscles.

The three-way interaction of the factors MUSCLE and VIBRATION CONDITION with either HS/HM $\left(F_{(4,40)}=87.6\right.$; $p<0.00001)$ or HM/MD $\left({ }^{\star} F_{(1.81,23.5)}=65.84 ; p<0.00001\right)$ as between-group factors were significant, which indicates that the effect of vibration on the hand muscles is significantly different in healthy subjects compared with healthy musicians, as well as in healthy musicians compared with musician's dystonia.

\section{Within-group effect of proprioceptive training on} sensorimotor organization

After proprioceptive training, the differential pattern of SMO was sharpened in healthy subjects: the effect of vibration on the vibrated (homotopic) and on the nonvibrated (heterotopic) muscles became stronger (Fig. 2). In healthy musicians, there was less cofacilitation of vibAPB or vibFDI on APB and FDI muscles after proprioceptive training, and the heterotopic effects of vibAPB on FDI and vibFDI on APB became stronger.

After proprioceptive training, the musician's dystonia patients showed a clear differential modulation in the effect of vibration on SICI. Although the homotopic effect was little changed, there was a clear increase of SICI in the nonvibrated muscles (heterotopic effects), which was strongest in muscles farther away from the vibrated one (e.g., SICI increase in ADM during vibAPB and vibFDI).

A three-way ANOVA performed with the factors TRAINING, MUSCLE, and VIBRATION CONDITION showed a significant three-way interaction in healthy subjects $\left(F_{(4,20)}=39.83 ; p<0.0001\right)$, in healthy musicians $\left(F_{(4,20)}=19.6 ; p<0.0001\right)$, and particularly in musician's dystonia patients $\left({ }^{\star} F_{(1.98,15.82)}=55.1 ; p<0.0001\right)$.

\section{Between-group effect of proprioceptive training on} sensorimotor organization

Table 2 displays the statistical results in detail. An ANOVA performed with VIBRATION CONDITION and MUSCLE as the within-group factor and HS/HM as the between-group factor showed significant interactions and main effects at baseline and after proprioceptive training. Therefore, the baseline differences in the effect of vibration on SICI in healthy subjects and healthy musicians persisted after proprioceptive training.

Using $\mathrm{HM} / \mathrm{MD}$ as the between-group factor, the three-way ANOVAs showed a significant three-way interaction and a sig- 


\begin{tabular}{|c|c|c|c|c|c|c|}
\hline & \multicolumn{3}{|c|}{ Three-way interaction } & \multicolumn{3}{|l|}{ Main effect } \\
\hline & $\mathrm{df} / \mathrm{error} \mathrm{df}$ & $F$ & $p$ & $\mathrm{df} /$ error df & $F$ & $p$ \\
\hline \multicolumn{7}{|c|}{$\mathrm{HS} / \mathrm{HM} \times$ muscle $\times$ vibration condition } \\
\hline HS after/HM after & $4 ; 48$ & 35.6 & $<0.0001$ & $1 ; 12$ & 21.2 & 0.001 \\
\hline \multicolumn{7}{|c|}{$\mathrm{HM} / \mathrm{MD} \times$ muscle $\times$ vibration condition } \\
\hline HM before/MD before & $1.81 ; 23.50^{*}$ & 65.84 & $<0.0001$ & $1 ; 14$ & 120.9 & $<0.0001$ \\
\hline HM after/MD after & $2.98 ; 38.69^{*}$ & 3.13 & 0.037 & $1 ; 14$ & 2.8 & 0.19 \\
\hline
\end{tabular}

Separate three-way ANOVAs were performed with either the data of HS/HM (top) or the data of HM/MD (bottom) as between-group factors and MUSCLE and VIBRATION CONDITION as within-group factors. Separate analyses were performed on the data obtained before or after the proprioceptive training. Furthermore, the data obtained in healthy musicians before and in musician's dystonia after proprioceptive training were compared. The $F$ and $p$ values for the three-way interaction and the main effect of the between-group factor are given. For ANOVAs with significant results $(p<0.05)$ in the Mauchly's test for sphericity, Greenhouse-Geisser corrections were performed, and corrected degrees of freedom (df) are given $(*)$.
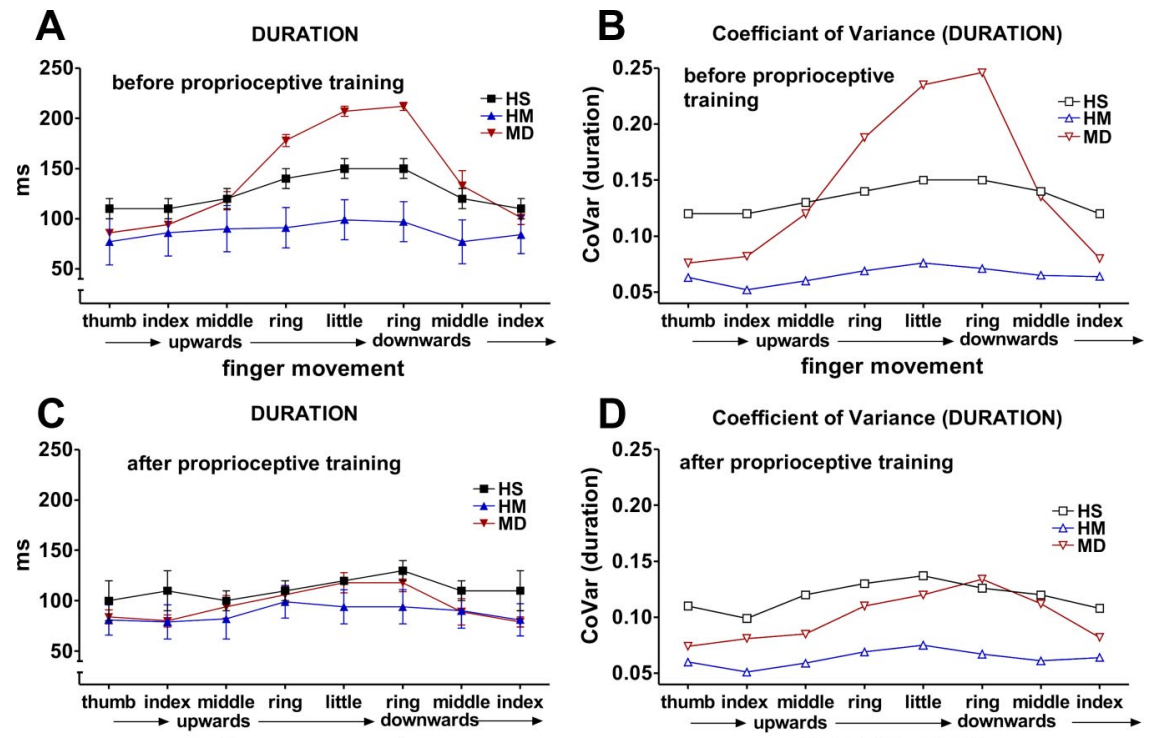

finger movement

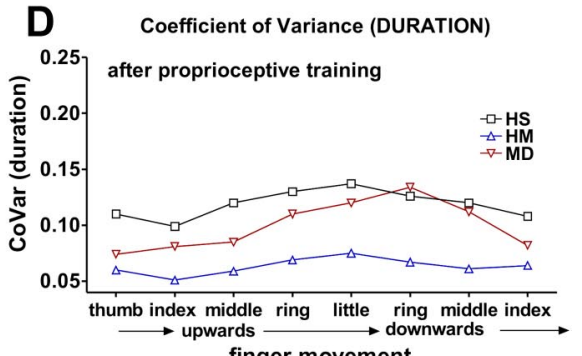

finger movement

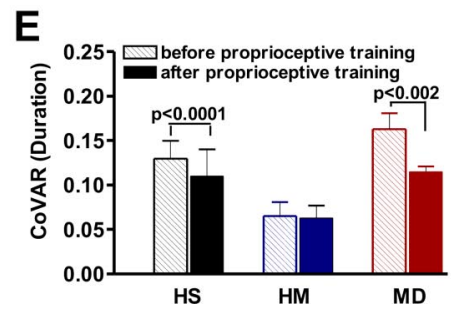

Figure 3. $\quad \boldsymbol{A}-\boldsymbol{D}$, The duration of key press ( $\boldsymbol{A}, \boldsymbol{C}$; left column) and the CoVar of the duration of key press $(\boldsymbol{B}, \boldsymbol{D}$; right column) for each finger movement of the test sequence, distinguishing between parts of the cycle when the finger was used in the ascending portion of the scale (thumb $\rightarrow$ index $\rightarrow$ middle $\rightarrow$ ring $\rightarrow$ little finger) or the descending portion (little finger $\rightarrow$ ring $\rightarrow$ middle $\rightarrow$ index $\rightarrow$ thumb). Subjects were asked to play staccato mezzoforte. The data obtained in HS (black), HM (blue), and MD (red) before $(\boldsymbol{A}, \boldsymbol{B}$; top) and after ( $\boldsymbol{C}, \boldsymbol{D}$; bottom) the proprioceptive training was given are shown. $\boldsymbol{E}$, The mean CoVar averaged over all finger movements before (left column) and after (right column) the proprioceptive training in HS, HM, and MD. Statistical results of paired $t$ tests comparing the data obtained before and after proprioceptive training are given. Error bars indicate SE.

nificant main effect of HM/MD only for the baseline data. For the data obtained after proprioceptive training, no interaction or main factor reached significance. Similarly there was no difference when comparing the data obtained in musician's dystonia after the proprioceptive training with those obtained in healthy musicians at baseline. In summary, these results show that the effect of vibration on SICI differed in healthy musicians and musician's dystonia at baseline; however, after proprioceptive training there was no significant difference between these two groups, which is likely attributable to the fact that the SMO in musician's dystonia became more differentially organized and thus more similar to that seen in healthy musicians.

\section{Piano performance data}

The detailed description, statistical analysis, and discussion of the piano performance data is performed on the data obtained during staccato playing. The data obtained during legato playing was similarly changed and is given in supplemental Figures 3 and 4 (available at www. jneurosci.org as supplemental material).

\section{Baseline performance parameters}

Figures 3 and 4 display the duration of key press (left column) and the CoVar of the duration of key press (right column) for each key press performed in the test sequence, distinguishing between upward (thumb to little finger) and downward (little finger to thumb) movements while the subjects were asked to play staccato mezzoforte (Fig. 3) or staccato pianissimo (Fig. 4).

Baseline piano performance before the application of proprioceptive training was quite different between the groups (Figs. $3 A, B, 4 A, B)$. As expected, the mean key press duration was generally shortest in healthy musicians in both performance conditions (mezzoforte and pianissimo) but showed also some slight prolongation in the ring and little finger. A one-way ANOVA with the factor FINGER MOVEMENT was significant for the duration data in both performance conditions $\left(F_{(7,35)}>6.8 ; p<0.001\right)$. However, the variability of performance (CoVar) was lowest in healthy musicians and did not show significant differences between single finger movements.

The musician's dystonia patients showed clear differences between the fingers, with the ring and little fingers showing the longest durations and the highest variability. Here, the oneway ANOVAs with the factor FINGER MOVEMENT on duration and CoVar (duration) data were significant in the mezzoforte and pianissimo conditions $\left({ }^{*} F_{(>2.08,>16.64)}>37.59\right.$; $p<0.0001)$.

Healthy (nonmusician) subjects showed longer key press durations, and the variability of performance (CoVar) was high, especially in the more difficult pianissimo condition (Fig. $4 A, B)$. There were slight performance differences between 
finger movements, with the "ring up," "little finger," and "ring down" finger movements showing the longest duration and the highest variability. However, this was not confirmed statistically since the one-way ANOVAs with the factor FINGER MOVEMENT was not significant in either performance condition for the duration of key press or the CoVar (duration).

\section{Within-group effect of proprioceptive} training on piano performance

Healthy musicians showed no change in their performance parameters after the proprioceptive training. A two-way ANOVA with the factors TRAINING and FINGER MOVEMENT revealed no significant interaction for either the duration or the CoVar (duration) data in both performance conditions (mezzoforte and pianissimo). Similar to their results at baseline, the duration of key press was slightly prolonged in the ring and little fingers (FINGER MOVEMENT; $F_{(7,35)}>3.2 ; p<$ 0.01 ), but there was no finger-movementspecific effect on the CoVar (duration).

In musician's dystonia patients, however, the prolonged duration and increased CoVar (duration) were clearly reduced after training, especially in the ring and little fingers. Two-way ANOVAs with the factors TRAINING and FINGER MOVEMENT showed a significant interaction for the duration and CoVar (duration) data, in the mezzoforte and pianissimo performance conditions $\left({ }^{*} F_{(>2.87,>22.95)}\right.$ $>9.79 ; p<0.0001)$ and significant main effects of the factor TRAINING $\left(F_{(1,8)}>23.4 ; p<0.002\right)$. In addition, the mean CoVar (duration) was significantly reduced after the proprioceptive training in both the mezzoforte and pianissimo performance conditions (Figs. $3 E, 4 E$ ) (paired $t$ tests, $p<0.001$ ).

In healthy subjects, piano performance appeared to be slightly improved after proprioceptive training with a reduction in the duration of key press and CoVar (duration). The two-way ANOVA with the factors TRAINING and FINGER MOVEMENT showed no significant interaction. However, there was a significant reduction of the mean CoVar (duration) (Fig. $3 E$ ) (paired $t$ test, $p<0.01)$ in the mezzoforte condition, indicating that piano performance was less variable after proprioceptive training.

Between-group comparison of the

effect of proprioceptive training

on piano performance

Before the proprioceptive training, the piano performance of musician's dystonia patients clearly differed from that of healthy musicians (Figs. $3 A, B, 4 A, B$ ). In the mezzoforte and pianissimo performance conditions, the duration of key press was longer in musician's dystonia, and the variability of performance higher. The difference was particularly striking in the ring and little fingers, which the patients had most difficulties in controlling. The two-way ANOVAs performed on the duration and CoVar (duration) data with HM/MD as the between-group factor and FINGER MOVEMENT as the within-group factor showed significant interactions for the mezzoforte and pianis-
DURATION
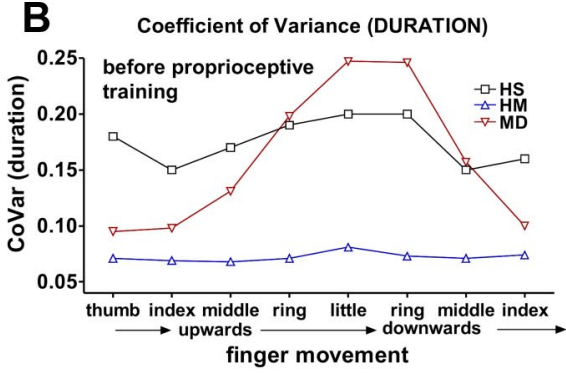

D Coefficient of Variance (DURATION)

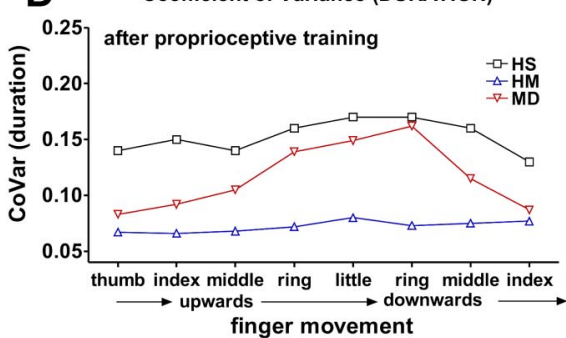

E

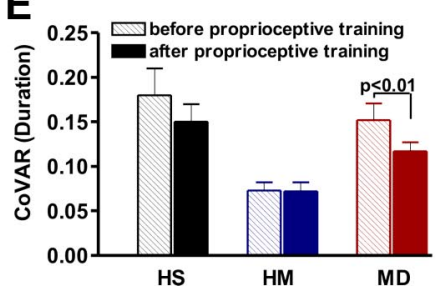

finger movement

Figure 4. The duration of key press and the CoVar of the duration of key press while subjects were asked to play staccato pianissimo. For additional details, refer to the legend of Figure 3 , since the layout is similar.

simo conditions, and also significant main effects of the factor HM/MD (see Table 3 for details of the statistics).

However, after the proprioceptive training, the performance in musician's dystonia patients changed and tended to become more like that of healthy musicians, particularly in the key press duration data. The same two-way ANOVAs as performed on the baseline performance data above were now calculated for the data after the proprioceptive training, and also on the data obtained in musician's dystonia after the training compared with those in healthy musicians before the training. In contrast to the findings in the baseline performance data, after the training there were neither significant interactions nor main effect of HM/MD for the duration of key press and CoVar (duration) in the mezzoforte or the pianissimo conditions (see Table 3 for details of the statistics). These statistical results confirm that particularly for the duration of key press, the performance of musician's dystonia patients after the proprioceptive training was more similar to that of healthy musicians either before or after the training.

Comparing the data of healthy musicians and nonmusicians, in the latter, in general, the duration of key press was longer and the variability higher. Before and after proprioceptive training, the twoway ANOVAs with HS/HM as the between-group factor and FINGER MOVEMENT as the within-group factor showed significant interactions for duration and CoVar (duration) in the mezzoforte and pianissimo conditions $\left(F_{(7,72)}<2.9: p<0.01\right)$, with HS/HM having a significant main effect $\left(F_{(1,12)}>13.5 ; p<0.001\right)$.

\section{Correlation of SMO and piano performance data in musician's dystonia}

Figure 5 displays the relationship between changes in SMO (SMOchange) and piano performance data (DURchange, 
Table 3. Statistical results of the two-way ANOVAs on the duration and CoVar (duration) data during piano performance

\begin{tabular}{|c|c|c|c|c|c|c|}
\hline & \multicolumn{3}{|c|}{ Two-way interaction: HM/MD $\times$ finger movement } & \multicolumn{3}{|c|}{ Main effect of HM/MD } \\
\hline & $\mathrm{df} /$ error df & $F$ & $p$ & df/error df & $F$ & $p$ \\
\hline \multicolumn{7}{|l|}{ Duration } \\
\hline \multicolumn{7}{|l|}{ MF } \\
\hline HM before/MD before & $2.36 ; 30.63^{*}$ & 18.79 & $<0.0001$ & $1 ; 14$ & 9.6 & 0.008 \\
\hline HM after/MD after & $2.39 ; 31.09^{*}$ & 3.64 & 0.031 & $1 ; 14$ & 0.1 & 0.71 \\
\hline HM before/MD after & $2.36 ; 30.70^{*}$ & 3.15 & 0.05 & $1 ; 14$ & 0.2 & 0.7 \\
\hline \multicolumn{7}{|l|}{ PP } \\
\hline HM before/MD before & $2.19 ; 28.51^{*}$ & 25.37 & $<0.0001$ & $1 ; 14$ & 34.8 & $<0.0001$ \\
\hline HM after/MD after & $3.42 ; 44.40^{*}$ & 4.39 & 0.007 & $1 ; 14$ & 5.5 & 0.179 \\
\hline \multicolumn{7}{|l|}{ CoVar } \\
\hline \multicolumn{7}{|l|}{ MF } \\
\hline HM before/MD before & $7 ; 98$ & 21.21 & $<0.0001$ & $1 ; 14$ & 122.7 & $<0.0001$ \\
\hline HM after/MD after & $3.58 ; 46.48^{*}$ & 2.67 & 0.049 & $1 ; 14$ & 91.8 & 0.016 \\
\hline HM before/MD after & $3.88 ; 50.50^{*}$ & 2.1 & 0.98 & $1 ; 14$ & 127.5 & 0.017 \\
\hline \multicolumn{7}{|l|}{$\mathrm{PP}$} \\
\hline HM before/MD before & $2.87 ; 37.31^{*}$ & 19.32 & $<0.0001$ & $1 ; 14$ & 140.3 & $<0.0001$ \\
\hline HM after/MD after & $2.48 ; 32.24^{*}$ & 5.11 & 0.008 & $1 ; 14$ & 34.6 & 0.014 \\
\hline HM before/MD after & $2.40 ; 31.23^{*}$ & 6.16 & 0.004 & $1 ; 14$ & 33.2 & 0.013 \\
\hline
\end{tabular}

Table showing two-way ANOVAs on the piano performancedata obtained in HM and MD with HM/MD as the between-group factor and FINGER MOVEMENT as the within-group factor for the parameter duration and CoVar in the mezzoforte (MF) and pianissimo (PP) performance conditions. Separate analyses were performed on the data obtained before or after the proprioceptive training. Furthermore, the data obtained in healthy musicians before and in musician's dystonia after proprioceptive training were compared. The $F$ and $p$ values for the two-way interaction and the main effect of HM/MD as the between-group factor are given. For ANOVAs with significant results ( $p<0.05$ ) in the Mauchly's test for sphericity, Greenhouse-Geisser corrections were performed, and corrected degrees of freedom (df) are given $(*)$.

COVARchange) in the eight musician's dystonia patients. The significant correlations between SMOchange (see Materials and Methods) in the heterotopic near (Fig. 5A) and far (Fig. 5B) effects with DURchange and COVARchange (see Materials and Methods) in the ring finger downward movement and little finger $(p<0.05)$ are displayed. These results show that the restoration of a heterotopic (near and far) inhibitory effect in the SMO is associated with an improvement of control of ring and little finger movements, which were most affected by the dystonic symptoms (see Fig. 5 for results of $r^{2}$ ).

\section{Effect of proprioceptive training on self-assessment and clinical scales}

Three of six healthy subjects and one of eight healthy musicians perceived their piano performance as slightly improved after the training. However, all musician's dystonia patients reported a significant subjective improvement of motor performance that, in some cases, lasted up to $24 \mathrm{~h}$. This was paralleled by a significant improvement in the BFM and TCS scores (for further details, see supplemental Table 4, available at www.jneurosci.org as supplemental material).

\section{Vibration discrimination data}

In all groups, discrimination was more difficult when there were smaller differences to the baseline vibration frequency; furthermore, there appeared to be clear differences between the groups, with musician's dystonia patients and also healthy nonmusicians performing significantly worse than healthy musicians, independent of the muscle to which vibration was applied. The individuals' discrimination ability was not correlated to either motor performance on the piano or SMO data. Details of the results and the statistics are provided in supplemental Figure 5 (available at www.jneurosci.org as supplemental material).

\section{Discussion}

In pianists with musician's dystonia, proprioceptive training restored a differential spatial pattern of SMO similar to that seen in healthy pianists and, crucially, led to an objective improvement of piano playing that was significantly correlated with the degree of sensorimotor reorganization. In healthy musicians and non- musicians, SMO was slightly more differentiated after proprioceptive training, but motor performance remained essentially unchanged. We hypothesize that motor control in musician's dystonia is regained through restoring a more differential SMO similar to the pattern seen in healthy musicians, which seems to be behaviorally beneficial and to enable motor control on the highest level.

\section{SMO and hand motor control}

SMO describes how short periods of hand muscle vibration affect MEPs and SICI in different hand muscles (Rosenkranz and Rothwell, 2003). We concentrate here on reporting changes in SICI as a parameter of $\mathrm{GABA}_{\mathrm{A}}$ receptor-mediated inhibition (MüllerDahlhaus et al., 2008), which is likely to represent changes in cortical circuits (DiLazzaro et al., 1998). In healthy subjects, input from one muscle facilitates motor output to that muscle ("homotopic" effect) by decreasing SICI, whereas it reduces motor output to other muscles ("heterotopic" effect) by increasing SICI. Low-amplitude muscle vibration predominantly excites Ia afferents (Roll et al., 1989) and produces proprioceptive input from a resting muscle that reliably resembles that of an ongoing contraction (Albert et al., 2006; Roll et al., 2009). Proprioceptive input directly activates area 4 of the motor cortex (Hore et al., 1976; Fromm et al., 1984; Huffmann and Krubitzer, 2001; Golaszewski et al., 2002; Burton et al., 2008) and is of particular relevance for motor learning (Pavlides et al., 1993). This makes the SMO particularly well suited as a measure of sensorimotor interactions in the human motor cortex that are relevant for hand motor control and motor learning.

In professional musicians, the (baseline) pattern of SMO is less differentially organized than in nonmusicians. In them, proprioceptive input from muscles that are functionally related, such as APB and FDI, facilitates motor output to these muscles, whereas it suppresses the output to functionally unrelated ones, such as ADM (Rosenkranz et al., 2005). This specific pattern is likely to have behavioral advantages since it may facilitate motor control of adjacent fingers (e.g., for the playing of fast passages).

However, in musician's dystonia patients, the reorganization of SMO goes one step further: spatial differentiation disappears; 


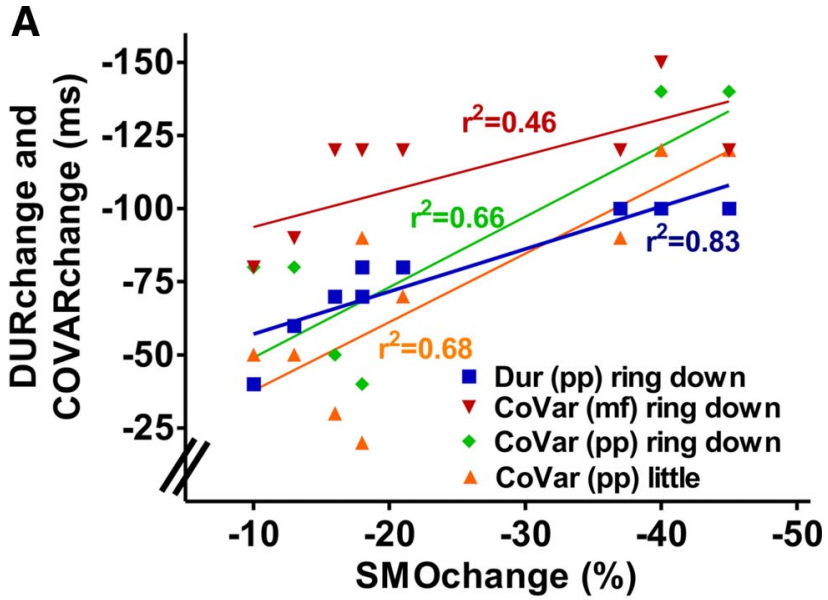

B

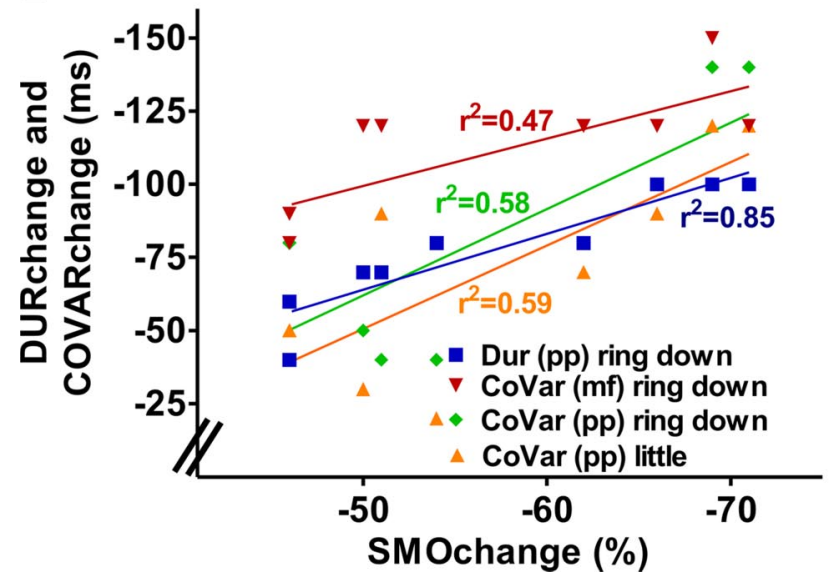

Figure 5. Correlation of the SMOChange and the changes in piano performance (DURchange and COVARchange) after proprioceptive training in the musician's dystonia patients. DURchange or COVARchange is calculated as the difference (after - before proprioceptive training) of the mean key press duration or CoVar (duration) for the ring finger downward and little finger movement for the mezzoforte and pianissimo conditions separately. These values are correlated to SMOchange, which is calculated as the difference (after - before proprioceptive training) of the normalized $\mathrm{SICl}$ data as displayed in Figure 3. $A$, The correlations with the near heterotopic effect averaged for the data obtained in APB and FDI. $\boldsymbol{B}$, Similarly for the far heterotopic effect. The $r^{2}$ values are given for the significant correlations $(p<0.05)$. The amount of change in the heterotopic near and far effects of $S \mathrm{MO}$ are both significantly correlated to performance improvement in the most affected ring finger downward movement in the five-finger-exercise.

instead, muscle vibration increases the excitability of projections to all hand muscles (Rosenkranz et al., 2005, 2008). Although this excess reorganization is associated with loss of task-specific motor control, it is not possible to say whether it causes the breakdown in motor control, or whether it is a consequence of persistent abnormal movement patterns. Several studies have considered a disorganization of sensorimotor integration, among other features, to be an "endophenotypic trait" in dystonia (Quartarone et al., 2006). Since, however, these features can also be induced in healthy humans by short- or long-term motor learning (Rosenkranz et al., 2006a, 2007b) and are found in highly trained professional musicians (Rosenkranz et al., 2007a), it cannot be excluded that they might represent an adaptation to dystonic movement patterns rather than being the cause of their development. In any case, alterations in cortical sensorimotor interactions are most likely to influence motor control, so that the association of SMO changes and the level of motor control is relevant regardless of its causality.

\section{Effect of proprioceptive training on SMO}

The way sensory representations are changed by sensory interventions depends on stimulation parameters such as attentional focus, timing, and location. Although synchronous stimulation leads to spatially separate representations being integrated or fused, asynchronous stimulation leads to separation (Xerri, 2008). In previous studies, we showed that similar factors determine the effect of interventions with proprioceptive stimulation on SMO in healthy human subjects (Rosenkranz and Rothwell, 2004, 2006b). Furthermore, in a comparative study on healthy nonmusicians, musicians, musician's dystonia patients, and writer's cramp patients, we found that the effect of proprioceptive interventions depends on the baseline pattern of SMO (Rosenkranz et al., 2008). Whereas the differential SMO in healthy subjects becomes undifferentiated by an intervention with vibration applied only to the $\mathrm{APB}$, the undifferentiated pattern seen in musician's dystonia regained some differentiation. Based on findings in the animal literature (Xerri, 2008), we modified the proprioceptive intervention and now applied vibration to three hand muscles randomly and asynchronously (one muscle at a time), with a concurrent discrimination task (proprioceptive training). We hypothesized that switching the "sensory input channel" and also the attentional focus would further sharpen the differential pattern of SMO in all groups. This reasoning was confirmed by the results. Proprioceptive training sharpened the differential profile of SMO particularly in the musician's dystonia patients. By strengthening the inhibitory heterotopic effects of proprioceptive input on the nonvibrated muscles, it restored a differential pattern of SMO similar to that seen in healthy musicians. In healthy musicians and nonmusicians, the effect of proprioceptive training was much weaker. Importantly, the slightly dedifferentiated baseline SMO of healthy musicians remained basically unchanged. We hypothesize that their SMO represents a stable and behaviorally beneficial pattern, established through long-term learning, which in contrast to the excessive SMO reorganization in musician's dystonia is not subject to short-term changes.

\section{Effect of proprioceptive training on piano performance}

Since studies of behavioral effects especially in patients often rely on investigator-dependent measures, such as clinical scales, special emphasis was given to use an objective measure of task-specific motor performance on the MIDI piano. Rather than a whole scale (Jabusch et al., 2004), we asked the subjects to play a five-finger exercise, which (1) was manageable by all subjects and (2) ensured an equal number of observations per finger movement for data analysis.

As expected, the performance of this five-finger exercise was worse in pianists with musician's dystonia than in healthy pianists, particularly when they used the ring and little fingers, whereas the performance of the other fingers (thumb, index, middle) was almost similar to healthy musicians.

Proprioceptive training had an immediate effect on performance in all musician's dystonia patients, which was seen best as a large reduction in variance of the ring and little finger movements to a level similar to that in the unaffected fingers. These findings support the reliability of the piano performance task in quantifying the degree and change of impairment, which is crucial for the assessment of any behavioral change (Spector and Brandfonbrener, 2007; Zeuner and Molloy, 2008). These objective results were paralleled by a substantial improvement in the patients' self-rating of performance, lasting for up to $24 \mathrm{~h}$, and in the significant changes in the BFM and TCS scores.

Proprioceptive training had no influence on piano performance in healthy musicians, whereas in healthy nonmusicians, 
whose piano performance was much worse than that of musicians, it tended to improve performance slightly. Additional control experiments would be needed to examine whether this was a within-session learning effect or whether proprioceptive training supported the consolidation of the newly learned motor skill.

\section{Does the sensorimotor reorganization induce the behavioral effect in musician's dystonia?}

A clear finding of this study is that restoring a more differential pattern of SMO was associated with improved motor performance on the piano in musician's dystonia. Furthermore, the degree of normalization of the heterotopic effect was significantly correlated to the decrease of key press duration and coefficient of variation in those finger movements, which were most affected in the patients. The immediate and strong effect of proprioceptive training is striking compared with training protocols using cutaneous stimulation (Zeuner et al., 2002, 2005) and might be attributable to differences in the central processing and integration of proprioceptive input in the motor cortex (see above).

All patients in this study had dystonia that involved "curling-in" of their ring (and little) finger, which is typical for pianists. These movements are produced mainly by contraction of superficial and deep finger flexors in the forearm, with minor contributions from the ADM, which flexes the proximal phalanx of the little finger. How could our proprioceptive training that involved only inputs from three hand muscles affect motor control of such distant muscles?

The most likely explanation relates to the fact that the motor cortex consists of a mosaic of intermingled output zones that each project to one or more different muscles (Schieber, 2002). Performance of discrete finger movements requires that particular spatial patterns of output are recruited within this mosaic. In patients with musician's dystonia, lack of differentiated SMO means that sensory input from one muscle, rather than facilitating the zones that control output to that muscle and inhibiting others, will facilitate wide output areas. This is likely to "defocus" any attempts to control spatial patterns of activity and will contribute to the symptoms of dystonia.

In this respect, we postulate that in musician's dystonia, the high variability in motor performance was caused by unfocussed integration of sensory feedback to motor output zones during finger movements. This interference may have been strongly reduced or no longer present after proprioceptive training increased GABAergic inhibition in motorcortical projections to nonvibrated muscles, thus allowing for more discrete movements to emerge.

\section{References}

Albert F, Bergenheim M, Ribot-Ciscar E, Roll JP (2006) The Ia afferent feedback of a given movement evokes the illusion of the same movement when returned to the subject via muscle tendon vibration. Exp Brain Res 172:163-174.

Altenmüller E (2003) Focal dystonia: advances in brain imaging and understanding of fine motor control in musicians. Hand Clin 19:523-538.

Bangert M, Schlaug G (2006) Specialization of the specialised in features of external human brain morphology. Eur J Neurosci 24:1832-1834.

Bengtsson SL, Nagy Z, Skare S, Forsman L, Forssberg H, Ullen F (2005) Extensive piano practicing has regionally specific effects on white matter development. Nat Neurosci 8:1148-1150.

Burton H, Sinclair RJ, McLaren DG (2008) Cortical network for vibrotactile attention: a fMRI study. Hum Brain Mapp 29:207-221.

Byl NN, Merzenich MM, Jenkins WM (1996) A primate genesis model of focal dystonia and repetitive strain injury: I. Learning-induced dedifferentiation of the representation of the hand in the primary somatosensory cortex in adult monkeys. Neurology 47:508-520.

Di Lazzaro V, Restuccia D, Oliviero A Profice P, Ferrara L, Insola A, Mazzone P, Tonali P, Rothwell JC (1998) Magnetic transcranial stimulation at intensities below active motor threshold activates intracortical inhibitory circuits. Exp Brain Res 119:265-268.

Elbert T, Pantev C, Wienbruch C, Rockstroh B, Taub E (1995) Increased cortical representation of the fingers of the left hand in string players. Science 270:305-307.

Elbert T, Candia V, Altenmuller E, Rau H, Sterr A, Rockstroh B, Pantev C, Taub E (1998) Alteration of digital representations in somatosensory cortex in focal hand dystonia. Neuroreport 6:3571-3575.

Fromm C, Wise SP, Evarts EV (1984) Sensory response properties of pyramidal tract neurons in the precentral motor cortex and postcentral gyrus of the rhesus monkey. Exp Brain Res 54:177-185.

Gaser C, Schlaug G (2003) Brain structures differ between musicians and non-musicians. J Neurosci 23:9240-9245.

Gilhodes JC, Roll JP, Tardy-Gervet MF (1986) Perceptual and motor effects of agonist-antagonist muscle vibration in man. Exp Brain Res 61:395-402.

Golaszewski SM, Siedentopf CM, Baldauf E, Koppelstaetter F, Eisner W, Unterrainer J, Guendisch GM, Mottaghy FM, Felber SR (2002) Functional magnetic resonance imaging of the human sensorimotor cortex using a novel vibrotactile stimulator. Neuroimage 17:421-430.

Hagbarth KE, Eklund G (1968) The effects of muscle vibration in spasticity, rigidity, and cerebellar disorders. J Neurol Neurosurg Psychiatry 31: 207-213.

Hore J, Preston JB, Cheney PD (1976) Responses of cortical neurons (areas 3a and 4) to ramp stretch of hindlimb muscles in the baboon. J Neurophysiol 39:484-500.

Huffmann KJ, Krubitzer L (2001) Thalamo-cortical connections of areas 3a and M1 in marmoset monkeys. J Comp Neurol 435:291-310.

Jabusch HC, Vauth H, Altenmüller E (2004) Quantification of focal dystonia in pianists using scale analysis. Mov Dis 19:171-180.

Kleim JA, Hogg TM, Vandenberg PM, Cooper NR, Bruneau R, Remple M (2004) Cortical synaptogenesis and motor map reorganization occur during late, but not early, phase of motor skill learning. J Neurosci 24:628-633.

Kujirai T, Caramia MD, Rothwell JC, Day BL, Thompson PD, Ferbert A, Wroe S, Asselman P, Marsden CD (1993) Corticocortical inhibition in human motor cortex. J Physiol 471:501-519.

Marsden CD, Meadows JC, Hodgson HJ (1969) Observations on the reflex response to muscle vibration in man and its voluntary control. Brain 92:829-846.

Müller-Dahlhaus JFM, Liu Y, Ziemann U (2008) Inhibitory circuits and the nature of their interaction in the human motor cortex: a pharmacological TMS study. J Physiol 586:495-514.

Nutt JG, Muenter MD, Melton LJ, Aronson A, Kurland LT (1988) Epidemiology of dystonia in Rochester, Minnesota. Adv Neurol 50:361-365.

Pavlides C, Miyashita E, Asanuma H (1993) Projections from the sensory to the motor cortex is important in learning motor skills in the monkey. J Neurophysiol 70:733-741.

Quartarone A, Siebner HR, Rothwell JC (2006) Task-specific hand dystonia: Can too much plasticity be bad for you? Trends Neurosci 29:192-199.

Roll JP, Gilhodes JC (1995) Proprioceptive sensory codes mediating movement trajectory perception: human hand vibration-induced drawing illusions. Can J Physiol Pharmacol 73:295-304.

Roll JP, Vedel JP, Ribot E (1989) Alteration of proprioceptive messages induced by tendon vibration in man: a microneurographic study. Exp Brain Res 76:213-222.

Roll JP, Albert F, Thyrion C, Ribot-Ciscar E, Bergenheim M, Mattei B (2009) Inducing any virtual two-dimensional movement in humans by applying muscle tendon vibration. J Neurophysiol 101:816-823.

Rosenkranz K, Rothwell JC (2003) Differential effect of muscle vibration on intracortical inhibitory circuits in humans. J Physiol 551:649-660.

Rosenkranz K, Rothwell JC (2004) The effect of sensory input and attention on the sensorimotor organization of the hand area of the human motor cortex. J Physiol 561:307-320.

Rosenkranz K, Rothwell JC (2006a) Differences between the effects of three plasticity inducing protocols on the organisation of the human motor cortex. Eur J Neurosci 23:822-829.

Rosenkranz K, Rothwell JC (2006b) Spatial attention affects sensorimotor re-organisation in human motor cortex. Exp Brain Res 170:97-108.

Rosenkranz K, Williamon A, Butler K, Cordivari C, Lees AJ, Rothwell JC (2005) Pathophysiological differences between musician's dystonia and writer's cramp. Brain 128:918-931. 
Rosenkranz K, Williamon A, Rothwell JC (2007a) Motorcortical excitability and plasticity is enhanced in professional musicians. J Neurosci 27:5200-5206.

Rosenkranz K, Kacar A, Rothwell J (2007b) Differential modulation of motor cortical plasticity and excitability in early and late phases of human motor learning. J Neurosci 27:12058-12066.

Rosenkranz K, Butler K, Williamon A, Cordivari C, Lees AJ, Rothwell JC (2008) Sensorimotor reorganisation by proprioceptive training in musician's dystonia and writer's cramp. Neurology 70:304-315.

Schieber MH (2002) Motor cortex and the distributed anatomy of finger movements. Adv Exp Med Biol 508:411-416.

Spector JT, Brandfonbrener AG (2007) Methods of evaluation of musician's dystonia: critique of measurement tools. Mov Dis 22:309-312.

Stinear CM, Byblow WD (2004) Elevated threshold for intracortical inhibition in focal hand dystonia. Mov Disord 19:1312-1317.
Tubiana R, Chamagne P (1993) Les affections professionelles du member supérieur chez les musicians. Bull Acad Natl Med 177:203-216.

Xerri C (2008) Imprinting of idiosyncratic experience in cortical snsory maps: Neural substrates of representational remodelling and correlative perceptual changes. Behav Brain Res 192:26-41.

Zeuner KE, Molloy FM (2008) Abnormal reorganisation in focal hand dystonia-sensory and motor training programs to retrain cortical function. NeuroRehabilitation 23:43-53.

Zeuner KE, Bara-Jimenez W, Noguchi PS, Goldstein SR, Dambrosia JM, Hallett M (2002) Sensory training for patients with focal hand dystonia. Ann Neurol 51:593-598.

Zeuner KE, Shill HA, Sohn YM, Molloy FM, Thornton BC, Dambrosia JM, Hallett M (2005) Motor training as treatment in focal hand dystonia. Mov Dis 20:335-341. 